\title{
Spin-3 quasinormal modes of BTZ black hole
}

\author{
Yun Soo Myung ${ }^{1, a}$, Yong-Wan Kim ${ }^{1, b}$, and Young-Jai Park ${ }^{2,3, c}$ \\ ${ }^{1}$ Institute of Basic Science and School of Computer Aided Science, \\ Inje University, Gimhae 621-749, Korea \\ ${ }^{2}$ Department of Physics and Center for Quantum Spacetime, \\ Sogang University, Seoul 121-742, Korea \\ ${ }^{3}$ Department of Service Systems Management and Engineering, \\ Sogang University, Seoul 121-742, Korea
}

\begin{abstract}
$\underline{\text { Abstract }}$
Using the operator approach, we obtain quasinormal modes (QNMs) of BTZ black hole in spin-3 topologically massive gravity by solving the first-order equation of motion with the transverse-traceless condition. We find that these are different from those obtained when solving the second-order differential equation for the third-rank tensor of spin-3 field subject to suitable boundary conditions and having the sign ambiguity of mass. However, it is shown clearly that two approaches to the left-moving QNMs are identical, while the rightmoving QNMs of solving the second-order equation are given by descendants of the operator approach.
\end{abstract}

PACS numbers: 04.70.Bw, 04.60.Kz, 04.30.Nk

Keywords: Quasinormal modes; BTZ black hole; spin-3 topologically massive gravity

${ }^{a}$ ysmyung@inje.ac.kr

${ }^{b}$ ywkim65@gmail.com

cyjpark@sogang.ac.kr 


\section{Introduction}

Recently, higher-spin theories on $\mathrm{AdS}_{3}$ have been paid much attention because they admit a truncation to an arbitrary maximal spin $N$ [1, 2]. Especially, the prototype of spin-3 model is a third-rank tensor of spin-3 field coupled to topologically massive gravity. The authors [3] have discussed the traceless spin-3 fluctuations around $\mathrm{AdS}_{3}$ spacetimes and, found that there exists a single massive propagating mode, besides left-moving and rightmoving massless modes (gauge artifacts). Also, a trace part of spin-3 fluctuations on $\mathrm{AdS}_{3}$ spacetimes has been studied in Ref. [4]. However, such a massive trace mode has zero energy and becomes pure gauge at the chiral point. These are considered through extended analysis of spin-2 field in the cosmological topologically massive gravity [5].

Very recently, Datta and David [6] have solved massive wave equations of arbitrary integer spin fields including spin-3 fields in the BTZ black hole background, and have obtained their quasinormal modes which are consistent with the location of the poles of the corresponding two-point function in the dual conformal field theory. This could be predicted by the $\mathrm{AdS}_{3} / \mathrm{CFT}_{2}$ correspondence. They have considered the second-order equation of $\left[\square-m^{2}+4 / \ell^{2}\right] \Phi_{\rho \mu \nu}=0$ for spin-3 fields with the ingoing modes at horizon and Dirichlet boundary condition at infinity. However, in this case, one confronts with sign ambiguity of mass $m$. Thus, in order to avoid this ambiguity, one could solve the first-order equation of $\epsilon_{\rho}^{\alpha \beta} \bar{\nabla}_{\alpha} \Phi_{\beta \mu \nu}+m \Phi_{\rho \mu \nu}=0$ itself with the transverse and traceless (TT) gauge condition.

On the other hand, it was known that the operator approach (method) [7] is very useful to derive the quasinormal modes of spin-2 fields in the non-rotating BTZ black hole background in the framework of cosmological topologically massive gravity. This method has been applied to new massive gravity to derive their quasinormal modes of the non-rotating BTZ black hole [8].

In this work, we obtain quasinormal modes of the non-rotating BTZ black hole in spin-3 topologically massive gravity by directly solving the first-order equation with the TT gauge condition in the operator approach. This method shows clearly how to derive quasinormal modes without sign ambiguity in mass.

\section{Perturbation analysis for spin-3 fields}

Since the spin-3 fluctuations on $\mathrm{AdS}_{3}$ or BTZ background was formulated in [3], let us write down the perturbation equation for the spin-3 fields $\Phi_{\mu \nu \lambda}=e_{\mu a b} \bar{e}_{\nu}^{a} \bar{e}_{\lambda}^{b}$ with $e_{\mu a b}$ spin-3 
connection and $\bar{e}_{\nu}^{a}$ the background dreibein as

$$
\bar{\square} \Phi^{\rho \alpha \beta}+\frac{1}{2 \mu} \epsilon^{\rho \mu \nu} \bar{\nabla}_{\mu} \bar{\square} \Phi_{\nu}^{\alpha \beta}=0 .
$$

Similar to the perturbed equation of the (spin-2) graviton,

$$
\left(\bar{\square}+\frac{2}{l^{2}}\right) h_{\sigma}^{\rho}+\frac{1}{\mu} \epsilon^{\rho \mu \nu} \bar{\nabla}_{\mu}\left(\bar{\square}+\frac{2}{l^{2}}\right) h_{\nu \sigma}=0,
$$

the spin-3 fluctuation also satisfies a third-order differential equation.

In this work, we consider the non-rotating BTZ black hole with the mass $M=1$ and the $\mathrm{AdS}_{3}$ curvature radius $\ell=1$ in global coordinates as

$$
d s_{\mathrm{BTZ}}^{2}=\bar{g}_{\mu \nu} d x^{\mu} d x^{\nu}=-\sinh ^{2} \rho d \tau^{2}+\cosh ^{2} \rho d \phi^{2}+d \rho^{2},
$$

where the event horizon is located at $\rho=0$, while the infinity is at $\rho=\infty$. Here we note that $\bar{g}_{\mu \nu}=\bar{e}_{\mu}^{a} \bar{e}_{\nu}^{b} \eta_{a b}$. In terms of the light-cone coordinates $u / v=\tau \pm \phi$, the metric tensor $\bar{g}_{\mu \nu}$ takes the form of

$$
\bar{g}_{\mu \nu}=\left(\begin{array}{ccc}
\frac{1}{4} & -\frac{1}{4} \cosh 2 \rho & 0 \\
-\frac{1}{4} \cosh 2 \rho & \frac{1}{4} & 0 \\
0 & 0 & 1
\end{array}\right) .
$$

Then the metric tensor (44) admits the Killing vector fields $L_{k}(k=0,-1,1)$ for the local $\mathrm{SL}(2, \mathrm{R}) \times \mathrm{SL}(2, \mathrm{R})$ algebra as

$$
L_{0}=-\partial_{u}, \quad L_{-1 / 1}=e^{\mp u}\left[-\frac{\cosh 2 \rho}{\sinh 2 \rho} \partial_{u}-\frac{1}{\sinh 2 \rho} \partial_{v} \mp \frac{1}{2} \partial_{\rho}\right],
$$

and $\bar{L}_{0}$ and $\bar{L}_{-1 / 1}$ are obtained by substituting $u \leftrightarrow v$. Locally, they form a basis of the $\mathrm{SL}(2, \mathrm{R})$ Lie algebra as

$$
\left[L_{0}, L_{ \pm 1}\right]=\mp L_{ \pm 1}, \quad\left[L_{1}, L_{-1}\right]=2 L_{0} .
$$

In the BTZ black hole background, the spin-3 field of $\Phi^{\rho \mu \nu}$ determined by a third-order differential equation (11) is totally symmetric and satisfies the TT gauge condition

$$
\Phi_{\mu}^{\mu \nu}=0, \quad \bar{\nabla}^{\mu} \Phi_{\mu \nu \rho}=0 .
$$

Hence its number of propagating degrees of freedom is counted to be one as

$$
10-3-6=1
$$

which corresponds to a single massive propagating mode as that in $\mathrm{AdS}_{3}$ background [3]. The third-order equation (11) can also be expressed as

$$
\left(\mathcal{D}^{M} \mathcal{D}^{L} \mathcal{D}^{R} \Phi\right)^{\rho \mu \nu}=0
$$


in terms of mutually commuting operators of

$$
\left(\mathcal{D}^{L / R}\right)^{\rho \nu}=\delta^{\rho \nu} \pm \frac{1}{2} \epsilon^{\rho \mu \nu} \bar{\nabla}_{\mu}, \quad\left(\mathcal{D}^{M}\right)^{\rho \nu}=\delta^{\rho \nu}+\frac{1}{2 \mu} \epsilon^{\rho \mu \nu} \bar{\nabla}_{\mu} .
$$

At the critical point of $\mu=1$, the operators $\mathcal{D}^{M}$ and $\mathcal{D}^{L}$ degenerate. We note that Eq. (9) is reduced to Eq. (1) when using the BTZ background

$$
\bar{R}_{\rho \sigma \mu \nu}=-\left(\bar{g}_{\rho \mu} \bar{g}_{\sigma \nu}-\bar{g}_{\rho \nu} \bar{g}_{\sigma \mu}\right), \quad \bar{R}_{\mu \nu=}=-2 \bar{g}_{\mu \nu},
$$

together with the TT gauge condition and the relation of $\left[\bar{\nabla}_{\mu}, \bar{\nabla}_{\nu}\right] \Phi^{\mu \alpha \beta}=-4 \Phi_{\nu}^{\alpha \beta}$. Therefore, the third-order equation (1) can be decomposed into three first-order differential equations:

$$
\left(\mathcal{D}^{M} \Phi\right)^{\rho \mu \nu}=0, \quad\left(\mathcal{D}^{L} \Phi\right)^{\rho \mu \nu}=0, \quad\left(\mathcal{D}^{R} \Phi\right)^{\rho \mu \nu}=0,
$$

for a massive, a left-moving, and a right-moving degree of freedom, respectively.

Importantly, three first-order differential equations (12) can be simply rewritten in terms of a single massive first-order differential equation as

$$
\epsilon_{\rho}^{\alpha \beta} \bar{\nabla}_{\alpha} \Phi_{\beta \mu \nu}+m \Phi_{\rho \mu \nu}=0
$$

with $m=2 \mu, 2$, and -2 . On the other hand, it could also be expressed in terms of a second-order differential equation [6] as

$$
\left[\square^{2}-m^{2}+4\right] \Phi_{\rho \mu \nu}=0
$$

At this stage, we wish to point out the presence of sign ambiguity $\pm m$ in the second-order equation (14). In order to avoid this ambiguity, one could directly solve the first-order equation (13) with the TT gauge condition.

Having the structure in mind, let us find quasinormal modes for the spin-3 field in the BTZ background by solving the equation of motion (13) with the TT gauge condition. In order to implement the operator method [7, 8], let us choose either the anti-chiral highest weight condition of $L_{1} \Phi_{\rho \mu \nu}=0$ or the chiral highest weight condition of $\bar{L}_{1} \Phi_{\rho \mu \nu}=0$, but not both simultaneously. Actually, we note that for a generic symmetric tensor $\Phi_{\rho \mu \nu}$, the transversality condition of $\bar{\nabla}^{\mu} \Phi_{\mu \nu \rho}=0$ is not equivalent to choosing the chiral (anti-chiral) highest weight condition.

\section{Left-moving quasinormal modes}

The least damped $(n=0)$ quasinormal mode can be found by considering the form

$$
\Phi_{\rho \mu \nu}(u, v, \rho)=e^{-i \omega \tau-i k \phi} F_{\rho \mu \nu}(\rho)=e^{-i h u-i \bar{h} v} F_{\rho \mu \nu}(\rho)
$$


with $\omega=h+\bar{h}$ and $k=h-\bar{h}$. This is the primary field which satisfies

$$
L_{0} \Phi_{\rho \mu \nu}(u, v, \rho)=i h \Phi_{\rho \mu \nu}(u, v, \rho), \quad \bar{L}_{0} \Phi_{\rho \mu \nu}(u, v, \rho)=i \bar{h} \Phi_{\rho \mu \nu}(u, v, \rho) .
$$

Note here that the subscript $\rho$ in $\Phi_{\rho \mu \nu}(u, v, \rho)$ is a dummy index, while $\rho$ in the argument is the radial coordinate in (3). It seems to be a formidable task to solve the first-order equation with the TT gauge condition without choosing a simplified form of $F_{\rho \mu \nu}$. Inspired by the lesson learned from the spin-2 analysis [7, 8], after tedious computations, we find the explicit solution

$$
\begin{aligned}
& F_{u \mu \nu}(\rho)=\left(\begin{array}{lll}
0 & 0 & 0 \\
0 & 0 & 0 \\
0 & 0 & 0
\end{array}\right), \\
& F_{v \mu \nu}(\rho)=\left(\begin{array}{ccc}
0 & 0 & 0 \\
0 & 1 & \frac{2}{\sinh 2 \rho} \\
0 & \frac{2}{\sinh 2 \rho} & \frac{4}{\sinh ^{2} 2 \rho}
\end{array}\right) F_{v v v}(\rho), \\
& F_{\rho \mu \nu}(\rho)=\left(\begin{array}{ccc}
0 & 0 & 0 \\
0 & \frac{2}{\sinh 2 \rho} & \frac{4}{\sinh ^{2} 2 \rho} \\
0 & \frac{4}{\sinh ^{2} 2 \rho} & \frac{8}{\sinh ^{3} 2 \rho}
\end{array}\right) F_{v v v}(\rho),
\end{aligned}
$$

which imply that $F_{v v v}(\rho)$ is a single massive propagating mode. Here Eq. (18) is similar to the spin-2 case [7], while Eqs. (17) and (19) represent new features of the spin-3 field.

Under the form of $\Phi_{\rho \mu \nu}(u, v, \rho)$ in Eq. (15) with $F_{\rho \mu \nu}(\rho)$ in Eqs. (17)-(19), the transversality condition of $\bar{\nabla}^{\mu} \Phi_{\mu \nu \rho}=0$ is equivalent to the anti-chiral highest weight condition of $L_{1} \Phi_{\rho \mu \nu}=0$, giving the constraint

$$
\sinh 2 \rho\left[\frac{d}{d \rho} F_{v v v}(\rho)\right]+2 i(\bar{h}+h \cosh 2 \rho) F_{v v v}(\rho)=0 .
$$

We emphasize that Eq. (20) takes the form of the equation for the scalar field $F_{v v v}(\rho)$, not a third-rank tensor field. Then, its solution is given by

$$
F_{v v v}(\rho)=C(\sinh 2 \rho)^{-i h}(\tanh \rho)^{-i \bar{h}}
$$

with a constant $C$. Finally, the equation of motion (13) determines $h$ as a function of $m$ of

$$
h=-i h_{L}(m), \quad h_{L}(m)=\frac{1}{2}(m-2) .
$$

Thus, the solution is summarized as

$$
\Phi_{\rho \mu \nu}^{L}(u, v, \rho)=e^{i k(\tau-\phi)-2 h_{L}(m) \tau} F_{\rho \mu \nu}^{L}(\rho)
$$


where $F_{\rho \mu \nu}^{L}(\rho)$ is given by Eqs. (17)-(19) with

$$
F_{v v v}^{L}(\rho)=(\sinh \rho)^{-2 h_{L}(m)}(\tanh \rho)^{i k} .
$$

Considering the form of quasinormal frequency

$$
\omega=\omega_{\mathrm{Re}}-i \omega_{\mathrm{Im}},
$$

we read off it from Eq. (23)

$$
\omega_{L}=-k-2 i h_{L}(m) .
$$

Thus, the solution (23) corresponds to a left-moving massive quasinormal mode of the least damped $(n=0)$ case for $m=2 \mu$, leading to

$$
h_{L}(\mu)=\mu-1>0 \quad \mu>1 .
$$

As is expected by the anti-chiral gravity, we observe that there is no quasinormal modes $\left(h_{L}=\right.$ 0 ) at the anti-chiral point of $\mu=1$. Also we observe that the asymptotic $\rho$-dependence of $F_{v v v}^{L}$ takes the form of $F_{v v v}^{L} \sim e^{2(1-\mu) \rho}$, which is compared to the spin-2 asymptotic dependence of $F_{v v} \sim e^{(1-\mu) \rho}$ for $m=\mu[9]$.

In order to derive the higher-order quasinormal modes, we act on the anti-chiral highest weight quasinormal modes with the operator of $\bar{L}_{-1} L_{-1}$. The effect of this will be to replace $\omega_{\text {Im }}$ by $\omega_{\operatorname{Im}}+2$ in Eq. (23). Hence one could expect to have

$$
\Phi_{\rho \mu \nu}^{(n) L}(u, v, \rho)=\left(\bar{L}_{-1} L_{-1}\right)^{n} \Phi_{\rho \mu \nu}^{L}(u, v, \rho),
$$

which are descendents of $\Phi_{\rho \mu \nu}^{L}(u, v, \rho)$. Since $\bar{L}_{-1} L_{-1}$ commutes with the equation (13), $\Phi_{\rho \mu \nu}^{(n) L}(u, v, \rho)$ is again the solution to the first-order equation with the same boundary condition of asymptotic fall-off as in $\Phi_{\rho \mu \nu}^{L}(u, v, \rho)$. Hence, the complete tower of the left-moving spin-3 quasinormal modes could be generated from $\Phi_{\rho \mu \nu}^{L}(u, v, \rho)$.

Consequently, the corresponding quasinormal frequencies are given by

$$
\omega_{L}^{n}=-k-2 i\left(h_{L}(\mu)+n\right), \quad n \in Z
$$




\section{Right-moving quasinormal modes}

On the other hand, right-moving quasinormal modes of the least damped case can be obtained by substitution of $u \rightarrow v, h \rightarrow \bar{h}$, and $m \rightarrow-m$. Explicitly, they take the form of

$$
\begin{aligned}
& F_{u \mu \nu}(\rho)=\left(\begin{array}{ccc}
1 & 0 & \frac{2}{\sinh 2 \rho} \\
0 & 0 & 0 \\
\frac{2}{\sinh 2 \rho} & 0 & \frac{4}{\sinh ^{2} 2 \rho}
\end{array}\right) F_{\text {uuu }}(\rho), \\
& F_{v \mu \nu}(\rho)=\left(\begin{array}{ccc}
0 & 0 & 0 \\
0 & 0 & 0 \\
0 & 0 & 0
\end{array}\right) \\
& F_{\rho \mu \nu}(\rho)=\left(\begin{array}{ccc}
\frac{2}{\sinh 2 \rho} & 0 & \frac{4}{\sinh ^{2} 2 \rho} \\
0 & 0 & 0 \\
\frac{4}{\sinh ^{2} 2 \rho} & 0 & \frac{8}{\sinh ^{3} 2 \rho}
\end{array}\right) F_{\text {uuu }}(\rho),
\end{aligned}
$$

which imply that $F_{\text {uuu }}(\rho)$ is a single massive propagating mode. Here Eq. (30) is similar to the spin-2 case [7], while Eqs. (31) and (32) represent new features of the spin-3 field.

The transversality condition of $\bar{\nabla}^{\mu} \Phi_{\mu \nu \rho}=0$ in this case is now compatible with the chiral highest weight condition of $\bar{L}_{1} \Phi_{\rho \mu \nu}=0$, giving the differential equation of $F_{\text {uuu }}(\rho)$

$$
\sinh 2 \rho\left[\frac{d}{d \rho} F_{\text {uиu }}(\rho)\right]+2 i(h+\bar{h} \cosh 2 \rho) F_{\text {uиu }}(\rho)=0 .
$$

The solution is given by

$$
F_{\text {uuи }}(\rho)=D(\sinh 2 \rho)^{-i \bar{h}}(\tanh \rho)^{-i h}
$$

with a constant $D$. The equation of motion (13) determines $\bar{h}$ as a function of $m$

$$
\bar{h}=-i h_{R}(m), \quad h_{R}(m)=\frac{1}{2}(m-2) .
$$

Considering $m=-2 \mu$, one finds

$$
h_{R}(\mu)=-\mu-1 \equiv \tilde{\mu}-1>0, \quad \mu<-1(\tilde{\mu}>1) .
$$

Then, the $n=0$ least damped right-moving solution is given by

$$
\Phi_{\rho \mu \nu}^{R}(u, v, \rho)=e^{-i k(\tau+\phi)-2 h_{R}(\mu) \tau} F_{\rho \mu \nu}^{R}(\rho)
$$

where $F_{\rho \mu \nu}^{R}(\rho)$ is given by Eqs. (301)-(32) with

$$
F_{\text {uиu }}^{R}(\rho)=(\sinh \rho)^{-2 h_{R}(m)}(\tanh \rho)^{-i k} .
$$


Thus, its quasinormal mode can be read off as

$$
\omega_{R}=k-2 i h_{R}(\mu)
$$

As is expected by the chiral gravity, we observe that there is no quasinormal modes $\left(h_{R}=0\right)$ at the chiral point of $\mu=-1$.

Similarly, the higher-order quasinormal modes are obtained by acting the operator of $\bar{L}_{-1} L_{-1}$ as

$$
\Phi_{\rho \mu \nu}^{(n) R}(u, v, \rho)=\left(\bar{L}_{-1} L_{-1}\right)^{n} \Phi_{\rho \mu \nu}^{R}(u, v, \rho),
$$

which are descendants of $\Phi_{\rho \mu \nu}^{R}(u, v, \rho)$. Its quasinormal frequencies are given by

$$
\omega_{R}^{n}=k-2 i\left(h_{R}(\mu)+n\right) .
$$

In Table 1, we have briefly summarized the results by comparing the spin-2 field in Ref. [7, 8] with the spin-3 topologically massive gravity. For the spin-2 field satisfying (2), the QNMs for the left-moving component exist for only $\mu>1$, while the QNMs for the rightmoving one for only $\mu<-1$ as in Ref. [7, 8]. Especially, the result of Ref. [7] is obtained by $u \rightarrow v, h \rightarrow \bar{h}$, but not by $m \rightarrow-m$. Instead, the authors gave the mass ranges for the QNMs as the left-moving (right-moving) component for $\mu>1(\mu<-1)$, which are exactly the same with replacing $m$ by $-m$ in the equation of motion as shown in Table. 1 . The QNMs for the left-moving (right-moving) spin-3 field are, by the same token, valid for only $\mu>1$ $(\mu<-1)$. Here we also note that there are no QNMs at $\mu=1(\mu=-1)$ for the left-moving (right-moving) spin-2 field, while at $\mu=1(\mu=-1)$ for the left-moving (right-moving) spin-3 field, expected by anti-chiral (chiral) gravity, respectively.

\section{Discussions}

We have obtained quasinormal modes of BTZ black hole in spin-3 topologically massive gravity by directly solving the first-order equation with the transverse-traceless condition in the operator approach. We have found that there is no $n=0$ quasinormal modes $\left(h_{L / R}=0\right)$ at the anti-chiral/chiral point of $\mu= \pm 1$.

It seemed that these are different from those with $T_{L / R}=\frac{1}{2 \pi}[6]$

$$
\omega_{s L}^{n}=k-2 \pi T_{L} i(2 n+m+1-s), \quad \omega_{s R}^{n}=-k-2 \pi T_{R} i(2 n+m+1+s),
$$

which are obtained when solving the second-order differential equations for the $s$-rank tensor of spin- $s$ field imposed by the boundary conditions. We note that the signs \pm of real part 


\begin{tabular}{cc}
\hline \hline \multicolumn{3}{c}{ Solutions of first-order differential equations } \\
\hline \multicolumn{2}{c}{ spin-2 field } \\
\hline$\epsilon_{\mu}^{\alpha \beta} \bar{\nabla}_{\alpha} h_{\beta \nu}+m h_{\mu \nu}=0$ & $\epsilon_{\mu}^{\alpha \beta} \bar{\nabla}_{\alpha} h_{\beta \nu}-m h_{\mu \nu}=0$ \\
$L_{1} h_{\mu \nu}=0$ & $\bar{L}_{1} h_{\mu \nu}=0$ \\
$h_{L}(m)=\frac{m}{2}-\left.\frac{1}{2}\right|_{m=\mu}=\frac{\mu}{2}-\frac{1}{2}$ & $h_{R}(m)=\frac{m}{2}-\left.\frac{1}{2}\right|_{m=-\mu}=-\frac{\mu}{2}-\frac{1}{2}$ \\
$\omega_{n}^{L}=-k-2 i\left(h_{L}(m)+n\right)$ & $\omega_{n}^{R}=k-2 i\left(h_{R}(m)+n\right)$ \\
\hline \multicolumn{2}{c}{ spin-3 field } \\
\hline$\epsilon_{\rho}^{\alpha \beta} \bar{\nabla}_{\alpha} \Phi_{\beta \mu \nu}+m \Phi_{\rho \mu \nu}=0$ & $\epsilon_{\rho}^{\alpha \beta} \bar{\nabla}_{\alpha} \Phi_{\beta \mu \nu}-m \Phi_{\rho \mu \nu}=0$ \\
$L_{1} \Phi_{\rho \mu \nu}=0$ & $\bar{L}_{1} \Phi_{\rho \mu \nu}=0$ \\
$h_{L}(m)=\frac{m}{2}-\left.1\right|_{m=2 \mu}=\mu-1$ & $h_{R}(m)=\frac{m}{2}-\left.1\right|_{m=-2 \mu}=-\mu-1$ \\
$\omega_{n}^{L}=-k-2 i\left(h_{L}(m)+n\right)$ & $\omega_{n}^{R}=k-2 i\left(h_{R}(m)+n\right)$ \\
\hline \hline
\end{tabular}

Table 1: Summary of the QNMs by comparing the spin-2 field in Ref. [8] with the spin-3 field in topologically massive gravity: The right-moving solution is obtained by solving the first-order equation of motion with the replacement of $u \rightarrow v, h \rightarrow \bar{h}$, and $m \rightarrow-m$. The equation of motion with $m$ allows only the left-moving (anti-chiral) solution, while the one with $-m$ gives only the right-moving (chiral) solution in Ref. [8].

$\omega_{s L / R}^{n}$ are different from $\mp$ of $\omega_{L / R}^{n}$. We adhere to the convention of Ref .[7] for the left/rightmoving modes. Comparing the left-moving QNMs in Eq. (29) with $\omega_{3 L}^{n}$ with $m=2 \mu$ leads to the same expression for the imaginary sector. The same thing happens for the spin-2 when comparing $\omega_{2 L}^{n}$ with $m=\mu$.

On the other hand, it seems that the right-moving QNMs in Eq. (41) are different from $\omega_{3 R}^{n}$. However, $\omega_{3 R}^{n}$ cloud be recovered from the descendants of $\Phi_{\rho \mu \nu}^{R}(u, v, \rho)[9$. That is, one has that imaginary $\left[\omega_{3 R}^{n}\right]=$ imaginary $\left[\omega_{R}^{n+3}\right]$ for spin-3. Similarly, one has that imaginary $\left[\omega_{2 R}^{n}\right]=$ imaginary $\left[\omega_{R}^{n+2}\right]$ for spin- 2 . We have constructed these descendants for the spin-2 case in Appendix A and the spin-3 case in Appendix B, whose asymptotic forms of relevant part are consistent with those obtained by solving the second-order differential equations.

For $\mu= \pm 1$, we expect to develop logarithmic modes of spin-3 field in the BTZ black hole background as did for spin-2 field [8, 10].

Consequently, the operator approach combined with the first-order equation is a useful method to derive QNMs of the BTZ black hole in the spin- $s$ topologically massive gravity. We suggest that two approaches to the left-moving QNMs are identical, while the right-moving QNMs of solving second-order equation are given by descendants of the operator approach. 


\section{Acknowledgement}

Two of us (Y. S. Myung and Y.-W. Kim) were supported by the National Research Foundation of Korea (NRF) grant funded by the Korea government (MEST) (No.2011-0027293). Y.-J. Park was partially supported by the National Research Foundation of Korea (NRF) grant funded by the Korea government (MEST) through the Center for Quantum Spacetime (CQUeST) of Sogang University with grant number 2005-0049409, and was also supported by World Class University program funded by the Ministry of Education, Science and Technology through the National Research Foundation of Korea(No. R31-20002).

\section{Appendix: Descendants of spin- $s$ field}

\section{A. Descendants of spin-2 field}

It was known that by solving the first-order equation of $\epsilon_{\mu}{ }^{\alpha \beta} \bar{\nabla}_{\alpha} h_{\beta \nu}+m h_{\mu \nu}=0$ with the TT condition, one has the ingoing highest weight solution for the left-moving spin-2 field near the horizon [ㅇ, 9]

$$
h_{\mu \nu}^{L}=e^{(1-\mu) t+i k(t-\phi)}(\sinh \rho)^{1-\mu}(\tanh \rho)^{i k}\left(\begin{array}{ccc}
0 & 0 & 0 \\
0 & 1 & \frac{2}{\sinh 2 \rho} \\
0 & \frac{2}{\sinh 2 \rho} & \frac{4}{\sinh ^{2} 2 \rho}
\end{array}\right) .
$$

On the other hand, by the substitution of $u \rightarrow v, h \rightarrow \bar{h}$, and $m \rightarrow-m$, we have the ingoing highest weight solution for the right-moving spin-2 field near the horizon

$$
h_{\mu \nu}^{R}=e^{(\mu+1) t-i k(t+\phi)}(\sinh \rho)^{\mu+1}(\tanh \rho)^{-i k}\left(\begin{array}{ccc}
1 & 0 & \frac{2}{\sinh 2 \rho} \\
0 & 0 & 0 \\
\frac{2}{\sinh 2 \rho} & 0 & \frac{4}{\sinh ^{2} 2 \rho}
\end{array}\right) .
$$

By acting the operator of $\bar{L}_{-1} L_{-1}$, the second descendent for the right-moving mode is given by

$$
\begin{aligned}
h_{\mu \nu}^{(2) R} & =\left(\bar{L}_{-1} L_{-1}\right)^{2} h_{\mu \nu}^{R} \\
& =\frac{1}{8} e^{-2 h_{R}(\mu) t-i k(t+\phi)}(\sinh \rho)^{\mu-3}(\tanh \rho)^{-i k}\left(\begin{array}{ccc}
m_{u u} & 0 & \frac{m_{u \rho}}{\sinh 2 \rho} \\
0 & 0 & 0 \\
\frac{m_{u \rho}}{\sinh 2 \rho} & 0 & \frac{m_{\rho \rho}}{\sinh ^{2} 2 \rho}
\end{array}\right)
\end{aligned}
$$


where

$$
h_{R}(\mu)=-\frac{\mu}{2}+\frac{3}{2}
$$

and

$$
\begin{aligned}
& m_{u u}=\left(\mu(1+\mu)-k^{2}-i k(1+2 \mu)\right) \times \\
& \left(16-13 \mu+3 \mu^{2}-8 k^{2}+4 \mu(-3+\mu) \cosh 2 \rho+\mu(1+\mu) \cosh 4 \rho\right. \\
& -8 i k(-3+\mu+\mu \cosh 2 \rho)) \text {, } \\
& m_{u \rho}=\frac{1}{8 \cosh ^{4} \rho}\left\{-16+40 \mu+139 \mu^{2}-150 \mu^{3}+35 \mu^{4}-k^{2}\left(172-475 \mu+243 \mu^{2}\right)\right. \\
& +48 k^{4}-i k\left(100+283 \mu-465 \mu^{2}+150 \mu^{3}+16 k^{2}(10-11 \mu)\right) \\
& +4\left(2(1-\mu)^{2}\left(-11-15 \mu+7 \mu^{2}\right)-k^{2}\left(39-181 \mu+90 \mu^{2}\right)+16 k^{4}\right. \\
& \left.+i k\left(7-102 \mu+177 \mu^{2}-58 \mu^{3}-62 k^{2}(1-\mu)\right)\right) \cosh 2 \rho \\
& +4\left(-2+6 \mu+21 \mu^{2}-26 \mu^{3}+7 \mu^{4}-k^{2}\left(25-73 \mu+35 \mu^{2}\right)+4 k^{4}\right. \\
& \left.-i k\left(15+41 \mu-75 \mu^{2}+26 \mu^{3}+4 k^{2}(6-5 \mu)\right)\right) \cosh 4 \rho \\
& \text { - } 4(1-\mu)\left(2\left(1-2 \mu^{2}+\mu^{3}\right)+k^{2}(5-6 \mu)-i k\left(1-9 \mu+6 \mu^{2}-2 k^{2}\right)\right) \cosh 6 \rho \\
& \left.+\mu(1-\mu)\left(\mu(1-\mu)+k^{2}-i k(1-2 \mu)\right) \cosh 8 \rho\right\} \text {, } \\
& m_{\rho \rho}=\frac{1}{4 \cosh ^{4} \rho}\left\{140-196 \mu+391 \mu^{2}-210 \mu^{3}+35 \mu^{4}-k^{2}\left(502-665 \mu+243 \mu^{2}\right)\right. \\
& +48 k^{4}-i k\left(14+823 \mu-651 \mu^{2}+150 \mu^{3}+16 k^{2}(14-11 \mu)\right) \\
& -8\left(28+44 \mu-77 \mu^{2}+42 \mu^{3}-7 \mu^{4}+k^{2}\left(62-133 \mu+45 \mu^{2}\right)-8 k^{4}\right. \\
& \left.-i k\left(86-153 \mu+129 \mu^{2}-29 \mu^{3}-k^{2}(46-31 \mu)\right)\right) \cosh 2 \rho \\
& +4\left(28-44 \mu+75 \mu^{2}-42 \mu^{3}+7 \mu^{4}-k^{2}\left(82-121 \mu+35 \mu^{2}\right)+4 k^{4}\right. \\
& \left.+i k(2-\mu)\left(1-71 \mu+26 \mu^{2}-20 k^{2}\right)\right) \cosh 4 \rho \\
& \text { - } 8(2-\mu)\left(2+3 \mu-4 \mu^{2}+\mu^{3}+k^{2}(5-3 \mu)-i k\left(5-9 \mu+3 \mu^{2}-k^{2}\right)\right) \cosh 6 \rho \\
& \left.+(1-\mu)(2+\mu)\left((1-\mu)(2-\mu)-k^{2}+i k(3-2 \mu)\right) \cosh 8 \rho\right\} \text {. }
\end{aligned}
$$

From $(\sinh \rho)^{(\mu-3)}$ in Eq. (45), its asymptotic form is given by

$$
h_{\rho \rho}^{(2) R} \sim e^{(\mu-3) \rho}, \quad \rho \rightarrow \infty,
$$

which is consistent with that of $n=0$ right-moving quasinormal modes obtained by solving the second-order differential equation [6]. Explicitly, from Eq. (3.55) in Ref. 6], we recover the same asymptotic form of $R_{22}(\xi) \sim e^{(m-3) \xi}$ for $\xi=\rho$ and $m=\mu$. 


\section{B. Descendants of spin-3 field}

As was done in the spin-2 field, from the right-moving highest weight solution for the spin-3 field in Eqs. (37) and (38), the third descendent quasinormal modes can be computed as

$$
\begin{aligned}
\Phi_{\rho \mu \nu}^{(3) R} & =\left(\bar{L}_{-1} L_{-1}\right)^{3} \Phi_{\rho \mu \nu}^{R} \\
& =\frac{1}{8} e^{-2 h_{R}(\mu) t-i k(t+\phi)}(\sinh \rho)^{2(\mu-2)}(\tanh \rho)^{-i k} F_{\rho \mu \nu}^{(3)}(\rho)
\end{aligned}
$$

where

$$
h_{R}(\mu)=-\mu+2 \text {, }
$$

and

$$
\begin{aligned}
F_{u \mu \nu}^{(3)}(\rho) & =\left(\begin{array}{ccc}
m_{u u u} & 0 & \frac{m_{u u \rho}}{\sinh 2 \rho} \\
0 & 0 & 0 \\
\frac{m_{u u \rho}}{\sinh 2 \rho} & 0 & \frac{m_{u \rho \rho}}{\sinh ^{2} 2 \rho}
\end{array}\right), \\
F_{v \mu \nu}^{(3)}(\rho) & =\left(\begin{array}{ccc}
0 & 0 & 0 \\
0 & 0 & 0 \\
0 & 0 & 0
\end{array}\right), \\
F_{\rho \mu \nu}^{(3)}(\rho) & =\left(\begin{array}{ccc}
\frac{m_{u u \rho} \rho}{\sinh 2 \rho} & 0 & \frac{m_{u \rho \rho}}{\sinh ^{2} 2 \rho} \\
0 & 0 & 0 \\
\frac{m_{u \rho \rho}}{\sinh ^{2} 2 \rho} & 0 & \frac{m_{\rho \rho \rho}}{\sinh ^{3} 2 \rho}
\end{array}\right)
\end{aligned}
$$

with

$$
\begin{aligned}
m_{\text {uиu }}= & \left\{(1+2 \mu)\left(4 \mu(1+\mu)-3 k^{2}\right)-i k\left(2\left(1+6 \mu+6 \mu^{2}\right)-k^{2}\right)\right\} \times \\
& \left\{2(-2+\mu)\left(12-19 \mu+10 \mu^{2}-12 k^{2}\right)-2 i k\left(44-51 \mu+18 \mu^{2}-4 k^{2}\right)\right. \\
& +3 \mu\left(29-33 \mu+10 \mu^{2}-8 k^{2}-16 i k(-2+\mu)\right) \cosh 2 \rho \\
& +6 \mu(-2+\mu-i k)(1+2 \mu) \cosh 4 \rho+\mu(1+\mu)(1+2 \mu) \cosh 6 \rho\}
\end{aligned}
$$




$$
\begin{aligned}
& m_{u u \rho}=\frac{1}{64 \cosh ^{6} \rho}\left\{84(1-2 \mu)^{2}\left(27+90 \mu-25 \mu^{2}-100 \mu^{3}+44 \mu^{4}\right)\right. \\
& +28 k^{2}\left(115-912 \mu-1485 \mu^{2}+4560 \mu^{3}-2172 \mu^{4}\right) \\
& -12\left(-6\left(9-42 \mu-523 \mu^{2}+700 \mu^{3}+636 \mu^{4}-1120 \mu^{5}+352 \mu^{6}\right)\right. \\
& +4 k^{2}\left(-278+819 \mu+1382 \mu^{2}-4340 \mu^{3}+2104 \mu^{4}\right) \\
& +k^{4}\left(-214+2242 \mu-2244 \mu^{2}\right)+40 k^{6} \\
& +i k\left(-525-3181 \mu+6468 \mu^{2}+7512 \mu^{3}-17080 \mu^{4}+6528 \mu^{5}\right. \\
& \left.\left.-k^{2}\left(477+1791 \mu-8822 \mu^{2}+5792 \mu^{3}\right)+4 k^{4}(-57+116 \mu)\right)\right) \cosh 2 \rho \\
& -3\left(-6(1-2 \mu)^{2}\left(108+336 \mu-169 \mu^{2}-420 \mu^{3}+220 \mu^{4}\right)\right. \\
& +2 k^{2}\left(-516+3296 \mu+5585 \mu^{2}-18560 \mu^{3}+9532 \mu^{4}\right) \\
& -16 k^{4}\left(29-282 \mu+276 \mu^{2}\right)+64 k^{6} \\
& +i k\left(576-6603 \mu+13888 \mu^{2}+14768 \mu^{3}-37760 \mu^{4}+15600 \mu^{5}\right. \\
& \left.\left.-k^{2}\left(1168+3739 \mu-18272 \mu^{2}+12308 \mu^{3}\right)+64 k^{4}(-7+13 \mu)\right)\right) \cosh 4 \rho \\
& -2\left(-36+216 \mu+2966 \mu^{2}-5400 \mu^{3}-2776 \mu^{4}+8640 \mu^{5}-3520 \mu^{6}\right. \\
& +4 k^{2}\left(-236+843 \mu+1302 \mu^{2}-4620 \mu^{3}+2664 \mu^{4}\right) \\
& -2 k^{4}\left(158-951 \mu+942 \mu^{2}\right)+16 k^{6} \\
& +i k\left(-474-2723 \mu+7188 \mu^{2}+6152 \mu^{3}-20040 \mu^{4}+9600 \mu^{5}\right. \\
& \left.\left.-k^{2}\left(498+2045 \mu-8442 \mu^{2}+6112 \mu^{3}\right)+24 k^{4}(-7+12 \mu)\right)\right) \cosh 6 \rho \\
& +6(-1+2 \mu)\left(2\left(-9+77 \mu^{2}-46 \mu^{3}-116 \mu^{4}+88 \mu^{5}\right)\right. \\
& +4 k^{2}\left(-46+36 \mu+358 \mu^{2}-404 \mu^{3}\right)+4 k^{4}(-4+11 \mu) \\
& -i k\left(-15-175 \mu-114 \mu^{2}-468 \mu^{3}+424 \mu^{4}\right. \\
& \left.\left.+k^{2}\left(1+123 \mu-190 \mu^{2}\right)+4 k^{4}\right)\right) \cosh 8 \rho \\
& +6 \mu(-1+2 \mu)\left(2 \mu\left(5-10 \mu-8 \mu^{2}+16 \mu^{3}\right)+k^{2}\left(4+16 \mu-48 \mu^{2}\right)+2 k^{4}\right. \\
& \left.+i k\left(-5+18 \mu+28 \mu^{2}-64 \mu^{3}+k^{2}(-3+16 \mu)\right)\right) \cosh 10 \rho \\
& \left.+\left(2 \mu^{2}\left(1-4 \mu^{2}\right)\left(1-4 \mu^{2}+3 k^{2}\right)+i k \mu\left(1-4 \mu^{2}\right)\left(1+12 \mu^{2}-k^{2}\right)\right) \cosh 12 \rho\right\}
\end{aligned}
$$




$$
\begin{aligned}
& m_{u \rho \rho}=\frac{1}{16 \cosh ^{6} \rho}\left\{2 \left(12\left(189+284 \mu-518 \mu^{2}-632 \mu^{3}+2037 \mu^{4}-1428 \mu^{5}+308 \mu^{6}\right)\right.\right. \\
& -k^{2}\left(1010-4695 \mu+37881 \mu^{2}-45384 \mu^{3}+15204 \mu^{4}\right) \\
& \left.+k^{4}\left(841-2991 \mu+2118 \mu^{2}\right)-80 k^{6}\right) \\
& -2 i k\left(2\left(2931-2812 \mu-5415 \mu^{2}+24814 \mu^{3}-22050 \mu^{4}+5796 \mu^{5}\right)\right. \\
& \left.+k^{2}\left(210-12953 \mu+23325 \mu^{2}-10678 \mu^{3}\right)+36 k^{4}(-17+25 \mu)\right) \\
& -12\left(630-752 \mu+1790 \mu^{2}+2108 \mu^{3}-6848 \mu^{4}+4848 \mu^{5}-1056 \mu^{6}\right. \\
& +k^{2}\left(-1167-1619 \mu+10438 \mu^{2}-12608 \mu^{3}+4208 \mu^{4}\right) \\
& +k^{4}\left(-433+1643 \mu-1122 \mu^{2}\right)+20 k^{6} \\
& +i k\left(-779-1969 \mu-3124 \mu^{2}+13816 \mu^{3}-12360 \mu^{4}+3264 \mu^{5}\right. \\
& \left.\left.+k^{2}\left(397-3487 \mu+6434 \mu^{2}-2896 \mu^{3}\right)+8 k^{4}(-21+29 \mu)\right)\right) \cosh 2 \rho \\
& -3\left(-4\left(360+448 \mu-1075 \mu^{2}-1198 \mu^{3}+4033 \mu^{4}-2940 \mu^{5}+660 \mu^{6}\right)\right. \\
& +k^{2}\left(272-3999 \mu+23723 \mu^{2}-28792 \mu^{3}+9532 \mu^{4}\right) \\
& -16 k^{4}\left(59-221 \mu+138 \mu^{2}\right)+32 k^{6} \\
& +i k\left(3536-4486 \mu-7450 \mu^{2}+32004 \mu^{3}-29100 \mu^{4}+7800 \mu^{5}\right. \\
& \left.\left.+k^{2}\left(496-7765 \mu+14255 \mu^{2}-6154 \mu^{3}\right)+32 k^{4}(-11+13 \mu)\right)\right) \cosh 4 \rho \\
& -2\left(810-1536 \mu+2434 \mu^{2}+2628 \mu^{3}-9728 \mu^{4}+7440 \mu^{5}-1760 \mu^{6}\right. \\
& +k^{2}\left(-949-1677 \mu+13818 \mu^{2}-16128 \mu^{3}+5328 \mu^{4}\right) \\
& +k^{4}\left(-599+1653 \mu-942 \mu^{2}\right)+8 k^{6} \\
& +i k(-1+\mu)\left(717+2212 \mu+6256 \mu^{2}-12600 \mu^{3}+4800 \mu^{4}\right. \\
& \left.\left.+k^{2}\left(-291+4318 \mu-3056 \mu^{2}\right)+144 k^{4}\right)\right) \cosh 6 \rho \\
& +6(-1+\mu)\left(4\left(-15-19 \mu+35 \mu^{2}+83 \mu^{3}-128 \mu^{4}+44 \mu^{5}\right)\right. \\
& -k^{2}\left(46+321 \mu-788 \mu^{2}+404 \mu^{3}\right)+k^{4}(-34+44 \mu) \\
& -i k\left(2\left(-59+101 \mu+272 \mu^{2}-518 \mu^{3}+212 \mu^{4}\right)\right. \\
& \left.\left.+k^{2}\left(-66+267 \mu-190 \mu^{2}\right)+4 k^{4}\right)\right) \cosh 8 \rho \\
& +6\left(1-3 \mu+2 \mu^{2}\right)\left(2\left(-3+7 \mu+4 \mu^{2}-16 \mu^{3}+8 \mu^{4}\right)+k^{2}\left(-5+28 \mu-24 \mu^{2}\right)+k^{4}\right. \\
& \left.+i k\left(-5-14 \mu+52 \mu^{2}-32 \mu^{3}+k^{2}(-5+8 \mu)\right)\right) \cosh 10 \rho \\
& +\left(\mu ( 1 - 3 \mu + 2 \mu ^ { 2 } ) \left(4 \mu\left(1-3 \mu+2 \mu^{2}\right)+3 k^{2}(1-2 \mu)\right.\right. \\
& \left.\left.+i k\left(-2+12 \mu-12 \mu^{2}+k^{2}\right)\right) \cosh 12 \rho\right\}
\end{aligned}
$$




$$
\begin{aligned}
& m_{\rho \rho \rho}=\frac{1}{16 \cosh ^{6} \rho}\left\{4 \left(3\left(3168+1488 \mu+3737 \mu^{2}-13896 \mu^{3}+15848 \mu^{4}-7392 \mu^{5}+1232 \mu^{6}\right)\right.\right. \\
& -k^{2}\left(9806-30354 \mu+74763 \mu^{2}-58848 \mu^{3}+15204 \mu^{4}\right) \\
& \left.+k^{4}\left(3362-7782 \mu+4236 \mu^{2}\right)-80 k^{6}\right) \\
& -2 i k\left(32556+26819 \mu-125136 \mu^{2}+194416 \mu^{3}-114240 \mu^{4}+23184 \mu^{5}\right. \\
& \left.+k^{2}\left(7920-51493 \mu+60576 \mu^{2}-21356 \mu^{3}\right)+12 k^{4}(-133+150 \mu)\right) \\
& +12\left(6\left(-1056+144 \mu+1003 \mu^{2}-3972 \mu^{3}+4516 \mu^{4}-2112 \mu^{5}+352 \mu^{6}\right)\right. \\
& -4 k^{2}\left(-575-4652 \mu+1043+\mu^{2}-8268 \mu^{3}+2104 \mu^{4}\right) \\
& +2 k^{4}\left(878-2165 \mu+1122 \mu^{2}\right)+40 k^{6} \\
& -i k\left(-7452+5081 \mu-36124 \mu^{2}+54952 \mu^{3}-32360 \mu^{4}+6528 \mu^{5}\right. \\
& \left.\left.+k^{2}\left(3696-14033 \mu+16914 \mu^{2}-5792 \mu^{3}\right)+k^{4}(-444+464 \mu)\right)\right) \cosh 2 \rho \\
& +3\left(6\left(2139+498 \mu+2407 \mu^{2}-9888 \mu^{3}+11224 \mu^{4}-5280 \mu^{5}+880 \mu^{6}\right)\right. \\
& -2 k^{2}\left(5561-22802 \mu+49529 \mu^{2}-39024 \mu^{3}+9532 \mu^{4}\right) \\
& +16 k^{4}\left(247-602 \mu+276 \mu^{2}\right)-64 k^{6} \\
& -i k\left(20475+13397 \mu-89648 \mu^{2}+133648 \mu^{3}-78640 \mu^{4}+15600 \mu^{5}\right. \\
& \left.\left.+k^{2}\left(6735-32439 \mu+38748 \mu^{2}-12308 \mu^{3}\right)+64 k^{4}(-15+13 \mu)\right)\right) \cosh 4 \rho \\
& -2\left(9486-5892 \mu-10870 \mu^{2}+38952 \mu^{3}-44776 \mu^{4}+21120 \mu^{5}-3520 \mu^{6}\right. \\
& +8 k^{2}\left(8-3141 \mu+7788 \mu^{2}-5754 \mu^{3}+1332 \mu^{4}\right) \\
& -2 k^{4}\left(1283-2355 \mu+942 \mu^{2}\right)+16 k^{6} \\
& +i k\left(-8913+15535 \mu-56124 \mu^{2}+86072 \mu^{3}-49560 \mu^{4}+9600 \mu^{5}\right. \\
& \left.\left.+k^{2}\left(4071-20363 \mu+21054 \mu^{2}-6112 \mu^{3}\right)+24 k^{4}(-17+12 \mu)\right)\right) \cosh 6 \rho \\
& +6(-3+2 \mu)\left(2\left(-114+4 \mu-165 \mu^{2}+538 \mu^{3}-396 \mu^{4}+88 \mu^{5}\right)\right. \\
& +k^{2}\left(80-956 \mu+1218 \mu^{2}-404 \mu^{3}\right)+k^{4}(-52+44 \mu) \\
& -i k\left(-260-205 \mu+1714 \mu^{2}-1604 \mu^{3}+424 \mu^{4}\right. \\
& \left.\left.+k^{2}\left(-184+411 \mu-190 \mu^{2}\right)+4 k^{4}\right)\right) \cosh 8 \rho \\
& +6\left(3-5 \mu+2 \mu^{2}\right)\left(2\left(-21+11 \mu+50 \mu^{2}-56 \mu^{3}+16 \mu^{4}\right)-8 k^{2}\left(5-12 \mu+6 \mu^{2}\right)\right. \\
& \left.+2 k^{4}+i k\left(7-130 \mu+180 \mu^{2}-64 \mu^{3}+k^{2}(-17+16 \mu)\right)\right) \cosh 10 \rho \\
& +\left(3-11 \mu+12 \mu^{2}-4 \mu^{3}\right)\left(6-22 \mu+24 \mu^{2}-8 \mu^{3}-6 k^{2}(1-\mu)\right. \\
& \left.\left.+i k\left(11-24 \mu+12 \mu^{2}-k^{2}\right)\right) \cosh 12 \rho\right\} \text {. }
\end{aligned}
$$

From $(\sinh \rho)^{2(\mu-2)}$ in Eq. (51), its asymptotic form is given by

$$
\Phi_{\rho \rho \rho}^{(3) R} \sim e^{2(\mu-2) \rho},
$$


which coincides with that of $n=0$ right-moving quasinormal modes obtained by solving the second-order differential equation [6]. Explicitly, from Eq. (B.32) in Ref. [6], we recover the same asymptotic form of $R_{222}(\xi) \sim e^{2\left(\frac{m}{2}-2\right) \xi}$ for $\xi=\rho$ and $m=2 \mu$.

\section{References}

[1] M. Henneaux and S. -J. Rey, JHEP 1012 (2010) 007 [arXiv:1008.4579 [hep-th]].

[2] A. Campoleoni, S. Fredenhagen, S. Pfenninger and S. Theisen, JHEP 1011 (2010) 007 arXiv:1008.4744 [hep-th]].

[3] B. Chen, J. Long and J. b. Wu, Phys. Lett. B 705 (2011) 513 [arXiv:1106.5141 [hep-th]].

[4] A. Bagchi, S. Lal, A. Saha and B. Sahoo, JHEP 1110 (2011) 150 arXiv:1107.0915 [hep-th]].

[5] W. Li, W. Song and A. Strominger, JHEP 0804 (2008) 082 [arXiv:0801.4566 [hep-th]].

[6] S. Datta and J. R. David, arXiv:1112.4619 [hep-th].

[7] I. Sachs and S. N. Solodukhin, JHEP 0808 (2008) 003 [arXiv:0806.1788 [hep-th]].

[8] Y. S. Myung, Y. W. Kim, T. Moon and Y. J. Park, Phys. Rev. D 84 (2011) 024044 arXiv:1105.4205 [hep-th]].

[9] D. Birmingham, S. Mokhtari and I. Sachs, Phys. Rev. D 82 (2010) 124059 arXiv:1006.5524 [hep-th]].

[10] I. Sachs, JHEP 0809 (2008) 073 [arXiv:0807.1844 [hep-th]]. 